\title{
Polyphenol and self-assembly: metal polyphenol nanonetwork for drug delivery and pharmaceutical applications
}

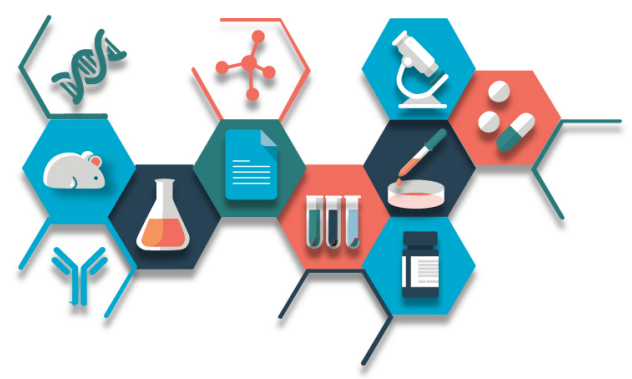

Xingcai Zhang*,1, Gaurav Parekh², Baoling Guo ${ }^{1,3}$, Xing Huang', Yuqing Dong', Wen Han $^{1}$, Xing Chen ${ }^{1}$ \& Gao Xiao ${ }^{1}$

${ }^{1}$ John A. Paulson School of Engineering \& Applied Sciences, Harvard University, Cambridge, MA 02138, USA

${ }^{2}$ Glanbia Nutritionals Inc., Twin Falls, ID 83301, USA

${ }^{3}$ Department of Clinical Oncology, Longyan First Hospital Affiliated to Fujian Medical University, Longyan, China, 364000

*Author for correspondence: xingcai@seas.harvard.edu

"One of the challenging aspects for nano-encapsulation or nano drug-delivery research has always been to scale up the production of such formulations at the industrial level."”

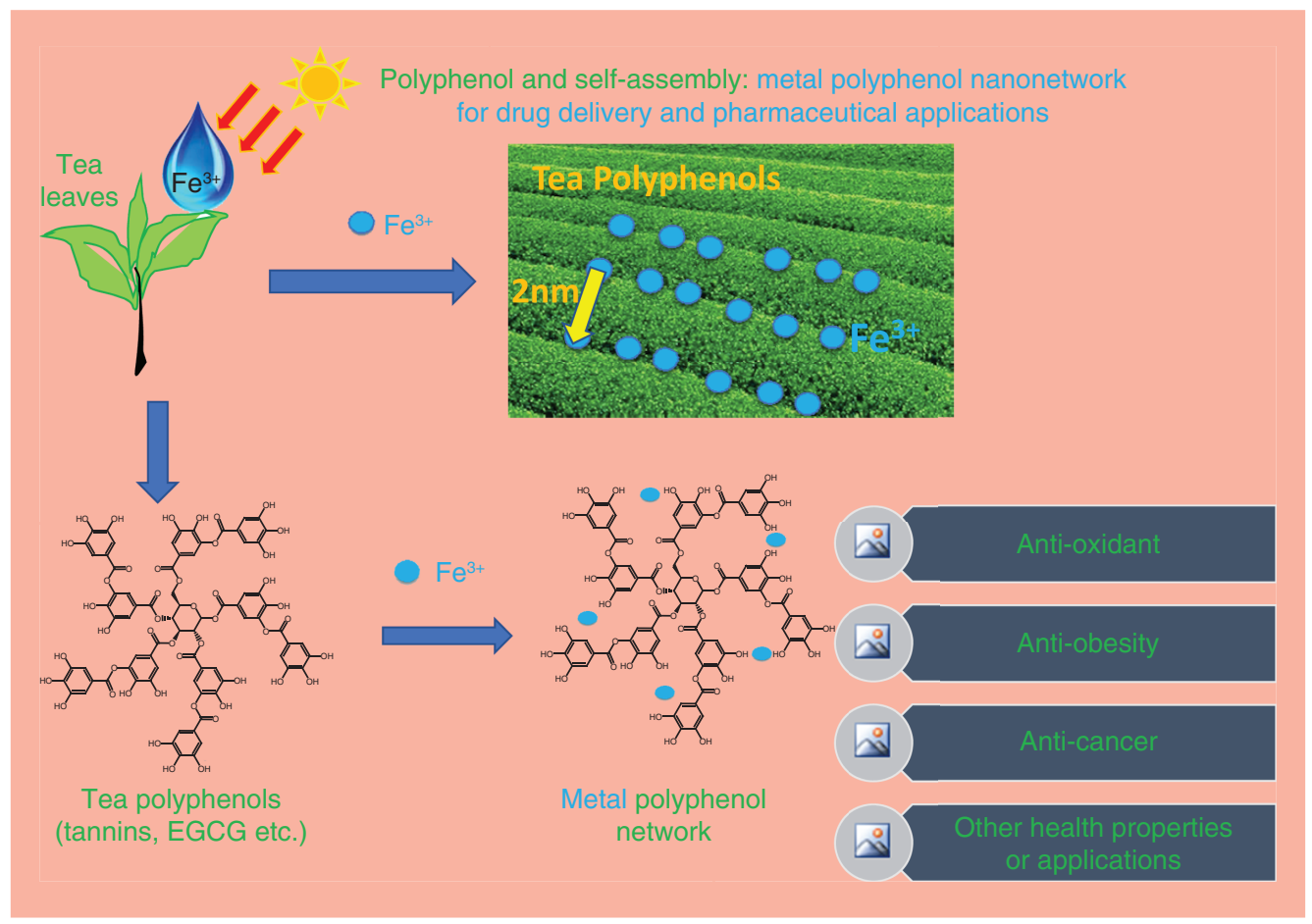

First draft submitted: 1 January 2019; Accepted for publication: 18 March 2019; Published online: 12 April 2019

Keywords: anti-cancer • bioavailability • drug delivery • metal polyphenol nanonetworks (MPN) • self-assembly • tea polyphenol • theranostics

Polyphenols have been a hot study area for the past few decades. Their antioxidant, antiobesity, anticancer and other health-related properties are major driving forces for their application in modern food, drug, dietary supplement, cosmetics and pharmaceutical industries. However, most polyphenols have limited bioavailability which confines their application. With the development of self-assembly technology, this problem can be solved. What is more, selfassembly technology has advanced to precisely control the thickness, morphology and functionalities of materials. 
Recently, polyphenols are found to be able to coordinate with metals and self-assemble into nanolayers with precious $2 \mathrm{~nm}$ thickness. Here, metal polyphenol nanonetwork (MPN) self-assembly technology and its various drug delivery and pharmaceutical applications are reviewed. Its challenges and prospects are discussed.

\section{Metal polyphenol nanonetworks}

Like most layer-by-layer self-assembly technology, MPNs cannot only grow as flat films but also take various forms due to their flexible structure. Besides, the functionality of MPN can be versatile based on the incorporated polyphenols and metal ions. The self-assembly of MPN films ( $\mathrm{Fe}^{3+}$-Tannic acid films) has been demonstrated using the layer-by-layer assembly technique. Shelf assembly can be used to control the thickness and morphology in both 2D and 3D structures [1-5]. Polyphenols have been defined as building blocks which can be engineered into versatile superstructures by varying the surface functionalization of its structure [6-9]. The ratio of catechol (C) and galloyl $(G)$ in the polyphenol structure determines their strength of binding to metal oxides, metal ions or even polymeric surfaces.

\section{Areas of biopharmaceutical applications}

Polyphenols have been studied and consumed both as food and supplements for decades, but the application of metal polyphenol nano-network (MPN) has been a new area of research. Its unique structure and property to form a thin film can be used for various applications. A combined crosslinked network of polyphenols with metal ions provide various applications based on the ratio of interaction. Tannic acid (TA) is one of the most common forms of polyphenols and had been widely applied for MPN research. A recent review [10] summarized the applications of different coating agents, along with tannic acid coordinate complexes, having different functionalities such as mechanical enhancing agent, $\mathrm{pH}$ responsive capsule, low-fouling agent, magnetic or image enhancer and enzyme immobilizer. It had also been shown that the coating of polyphenol-Fe shell on gold nanoparticle improved the catalytic property of the nanoparticle system [11]. For the past decade, with the versatility of the self-assembly technology due to the incorporation of different functional groups, precise control of nanometer thickness and nanoarchitecture, the amount of research in the area of using MPN for biopharmaceutical application has increased exponentially.

\section{Drug delivery carrier}

Due to the flexibility of the MPN films, they can take different shapes and can be widely used as a drug delivery carrier. In some research works, the single layer of coordinate Fe ${ }^{\mathrm{III}}$-TA films has been assembled on sacrificial template cores, such as on polystyrene template [12] or lignin nano/microparticle templates [13]. Similar one-step assembly of MPNs has been demonstrated using various dietary flavonoids coordinated to $\mathrm{Fe}^{\mathrm{III}}$ ions [14]. This way the health benefits of different flavonoids, such as anti-inflammatory, anti-cancer, antioxidant and antimicrobial, were exploited and water solubility improved by forming rapid self-assembly with metal ions in the form of films and capsules. MPNs with negative surface charge have been demonstrated to form quite a uniform multi-layer assembly in alteration with a positive polyelectrolyte, on polystyrene core [15]. Another piece of work shows that dopamine has been polymerized and assembled on silica templates. Here, the drug dopamine itself has been used as a phenolic film [16]. In other approaches, the core was loaded with drug/molecules and this core-template was dissolved to form hollow MPN capsules. Generally, MPNs dissolve at acidic $\mathrm{pH}$ and thus release the encapsulated $\mathrm{drug} / \mathrm{molecules}$. Some research works have demonstrated that polyphenols can form nanoparticle cores and then drugs can be loaded onto its surface by electrostatic interaction. Tea polyphenol nanoparticles (NP) can also be formed by oxidative polymerization [17]. Due to the low adhesion to blood vessel endothelium and selective adhesion to the myocardium, tannic acid was used to deliver proteins and peptides to the heart [18]. Most of the polyphenol $\mathrm{NP}$ is formed without any metal coordination. Incorporation of metal to the polyphenol matrix causes it to form films instead of NP. These examples demonstrate the vast application of MPNs as drug vehicles.

\section{Improving theranostic systems/cancer treatment}

Combinational therapy is a hot study area, especially for cancer treatment $[3,4]$ and MPNs has become a new star in the field. The property of polyphenol films binding readily at basic $\mathrm{pH}$ to heavy metal, for example, $\mathrm{Fe}^{\mathrm{III}}$ or $\mathrm{Fe}^{3+}$, $\mathrm{Gd}^{3+}$, etc. has been exploited for the theranostic antitumor therapy. Polyphenol network had been coordinately bonded to $\mathrm{Gd}^{3+}$ ion and assembled on a gold NP and they demonstrated a combinational antitumor therapy [19]. This provided an effective reduction in primary tumor volume due to photothermal effect due to gold NP and 
metastasis reduction due to polyphenols network. The $\mathrm{Gd}^{3+}$ ions enhanced the magnetic resonance imaging (MRI) and targeted delivery. In another work, prospects of TA-Fe ${ }^{\mathrm{III}}$ assembly on any nano-template have been validated for tumor-cell-specific photo-activated applications, such as near infra-red (NIR) photothermal antitumor therapy and as photoacoustic imaging along with MRI imaging [20]. TA crosslinked to collagen type I have been demonstrated to induce specific apoptosis of HER2 ${ }^{+}$breast cancer cell lines [21]. It has also been demonstrated that when polyphenol from green tea, epigallocatechin gallate (EGCG) coordinated with Fe $\mathrm{F}^{\mathrm{III}}$, was coated on therapeutic nanoformulations, it can lead to down-regulation of metastatic-associated factors. This, in turn, led to inhibition of epithelial-mesenchymal transition [22]. EGCG-Fe ${ }^{I I I}$-doxorubicin (DOX) nanocapsules have also shown the properties of responding to reactive oxygen species (ROS). Since tumor cells have higher ROS levels than systemic cells, EGCG-Fe ${ }^{I I I}$ nanocapsules demonstrated selective cytotoxicity to cancer cells with minimal systemic toxicity [23]. These nanocapsules are also $\mathrm{pH}$ sensitive. They release anticancer drugs, for example, DOX, at lower $\mathrm{pH}$ microenvironment where the polyphenol-metal ion coordinate bonds become weak at such low $\mathrm{pH}$ [24]. Since the extracellular microenvironment of tumor sides are as low as $\mathrm{pH} \sim 5.5$ [25], such a mechanism of drug release from MPN shell is promising. Research work demonstrated the functionalization of hyaluronic acid with phenol groups which, when coordinated with metal ion, formed synthetic MPN film [25]. They were coated on sacrificial $\mathrm{CaCO}_{3}$ templates to enhance adhesion to $\mathrm{CD}_{4} 4^{+}$of cancer cells, thus inhibiting their expression of anti-apoptosis protein synthesis [26]. Tuning the ratio of hyaluronic acid and polyethylene glycol (PEG) tails can alter the amount of MPN binding to $\mathrm{CD}_{4} 4^{+}$of cancer cells. A similar concept of PEG tails and low pH degradation of MPN was used, where the MPN film was assembled on nonsacrificial emulsion core made from oleic acid. The PEG tails acted as an anti-fouling agent against macrophages and the emulsion core helps to have higher encapsulation efficiency of the anti-cancer drug DOX [27].

\section{Future perspective \& steps}

The above research work shows that there are still a lot of untouched areas of biopharmaceutical applications for MPNs due to its facile procedure, versatility and precise nanoarchitecture buildup. Gene delivery has recently been an evolving area of research in the drug-delivery platform. From the above examples, it is clear that water-soluble drugs, for example, DOX can be delivered in the MPN nanocapsules. An earlier example, where MPN films were assembled over emulsion cores [27], opens up the area of encapsulating low soluble drugs in the emulsion, micelle, liposome or solid lipid nanoparticle cores. Even the approach of surface functionalization with varying MW proteins, for example, antibodies, and how it affects the MPN film properties can be a promising area of research and its application in targeted delivery.

The time to actually see such proof of concepts transition from lab scale to actual biopharmaceutical market has come. One of the challenging aspects for nano-encapsulation or nano drug-delivery research has always been to scale up the production of such formulations at the industrial level. The above areas should be the focus of future steps, but also a great deal of work is needed to finely tune the current most promising formulations to a production scale-up level.

Financial \& competing interests disclosure

The authors have no relevant affiliations or financial involvement with any organization or entity with a financial interest in or financial conflict with the subject matter or materials discussed in the manuscript. This includes employment, consultancies, honoraria, stock ownership or options, expert testimony, grants or patents received or pending, or royalties.

No writing assistance was utilized in the production of this manuscript.

\section{Open access}

This work is licensed under the Attribution-NonCommercial-NoDerivatives 4.0 Unported License. To view a copy of this license, visit http://creativecommons.org/licenses/by-nc-nd/4.0/

\section{References}

1. Rahim MDA, Kempe K, Müllner M et al. Surface-confined amorphous films from metal-coordinated simple phenolic ligands. Chem. Mater. 27(16), 5825-5832 (2015).

2. Zheng Z, Zhang X, Carbo D et al. Sonication-assisted synthesis of polyelectrolyte-coated curcumin nanoparticles. Langmuir 26(11), 7679-7681 (2010). 
3. Vergara D, Bellomo C, Zhang X et al. Lapatinib/Paclitaxel polyelectrolyte nanocapsules for overcoming multidrug resistance in ovarian cancer. Nanomedicine 8(6), 891-899 (2012).

4. Zhou M, Zhang X, Xie J et al. $\mathrm{pH}$-sensitive poly( $\beta$-amino ester)s nanocarriers facilitate the inhibition of drug resistance in breast cancer cells. Nanomaterials 8(11), 952 (2018).

5. Parekh G, Shi Y, Zheng J, Zhang X, Leporatti S. Nano-carriers for targeted delivery and biomedical imaging enhancement. Ther. Deliv. 9(6), 451-468 (2018).

6. Yang Y, Jin P, Zhang X et al. Improved anti-proliferative and pro-apoptotic effects of epigallocatechin gallate (EGCG) nanocomplexes co-assembled with 3- mercapto-1-hexanol and $\beta$-lactoglobulin. J. Biomed. Nanotechnol. 17, 1369 (2017).

7. Zhang X. Tea and cancer prevention. J. Can. Res. Updates 4(2), 65-73 (2015).

8. Tang P, Shen D, Xu Y, Zhang X, Shi J, Yin J. Effect of fermentation conditions and plucking standards of tea leaves on the chemical components and sensory quality of fermented juice. J. Chem. 4312875, 1-7 (2018).

9. Guo J, Tardy BL, Christofferson AJ et al. Modular assembly of superstructures from polyphenol-functionalized building blocks. Nat. Nanotechnol. 11(12), 1105-1111 (2016).

10. Ejima H, Richardson JJ, Caruso F. Metal-phenolic networks as a versatile platform to engineer nanomaterials and biointerfaces. Nano Today 12, 136-148 (2017).

11. Zeng T, Zhang X, Guo Y, Niu H, Cai Y. Enhanced catalytic application of Au@polyphenol-metal nanocomposites synthesized by a facile and green method. J. Mater. Chem. A 2(36), 14807-14811 (2014).

12. Ejima $\mathrm{H}$, Richardson JJ, Liang $\mathrm{K}$ et al. One-step assembly of coordination complexes for versatile film and particle engineering. Science 341(6142), 154-157 (2013).

13. Tardy BL, Richardson JJ, Guo J, Lehtonen J, Ago M, Rojas OJ. Lignin nano- and microparticles as template for nanostructured materials: formation of hollow metal-phenolic capsules. Green Chem. 20(6), 1335-1344 (2018).

14. Bertleff-Zieschang N, Rahim MDA, Ju Y et al. Biofunctional metal-phenolic films from dietary flavonoids. Chem. Commun. 53(6), 1068-1071 (2017).

15. Rahim MDA, Ejima H, Cho KL et al. Coordination-driven multistep assembly of metal-polyphenol films and capsules. Chem. Mater. 26(4), 1645-1653 (2014).

16. Ejima H, Richardson JJ, Caruso F. Phenolic film engineering for template-mediated microcapsule preparation. Polym. J. 46(8), 452-459 (2014).

17. Zhang H, Yi Z, Sun Z, Ma X, Li X. Functional nanoparticles of tea polyphenols for doxorubicin delivery in cancer treatment. J. Mater. Chem. B. 5(36), 7622-7631 (2017).

18. Shin M, Lee H-A, Lee M et al. Targeting protein and peptide therapeutics to the heart via tannic acid modification. Nat. Biomed. Eng. 2, 304-317 (2018).

19. Fan JX, Zheng DW, Mei WW et al. A metal-polyphenol network coated nanotheranostic system for metastatic tumor treatments. Small 13(48), 1702714 (2017).

20. Liu T, Zhang M, Liu Wet al. Metal ion/tannic acid assembly as a versatile photothermal platform in engineering multimodal nanotheranostics for advanced applications. ACS Nano 12(4), 3917-3927 (2018).

21. Reitzer F, Allais M, Ball V, Meyer F. Polyphenols at interfaces. Adv. Colloid Interface Sci. 257, 31-41 (2018).

22. Fan J-X, Zheng D-W, Rong L et al. Targeting epithelial-mesenchymal transition: metal organic network nano-complexes for preventing tumor metastasis. Biomaterials 139, 116-126 (2017).

23. Wang X, Li X, Liang X et al. ROS-responsive capsules engineered from green tea polyphenol-metal networks for anticancer drug delivery. J. Mater. Chem. B. 6(7), 1000-1010 (2018).

24. Ping $\mathrm{Y}$, Guo J, Ejima $\mathrm{H}$ et al. $\mathrm{pH}$-responsive capsules engineered from metal-phenolic networks for anticancer drug delivery. Small 11(17), 2032-2036 (2017).

25. Kato Y, Ozawa S, Miyamoto C et al. Acidic extracellular microenvironment and cancer. Cancer Cell Int. 13(1), 89-97 (2013).

26. Ju Y, Cui J, Sun H et al. Engineered metal-phenolic capsules show tunable targeted delivery to cancer cells. Biomacromolecules 17(6), 2268-2276 (2016).

27. Besford QA, Ju Y, Wang TY et al. Self-assembled metal-phenolic networks on emulsions as low-fouling and pH-responsive particles. Small 14(39), 1802342 (2018). 